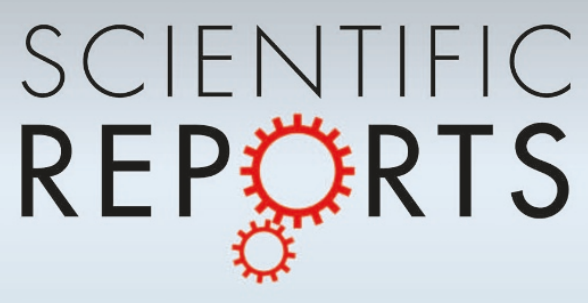

OPEN

SUBJECT AREAS: ANGIOGENESIS

NETWORKS AND SYSTEMS BIOLOGY

COMPUTATIONAL BIOLOGY

CANCER

Received

27 May 2011

Accepted

27 July 2011

Published

11 August 2011

Correspondence and requests for materials should be addressed to

M.Á.M. (medina@ uma.es)

* Present address: Institut de Biologia Evolutiva (CSIC-UPF), Passeig Marıtim de la Barceloneta 37-49, E-08003 Barcelona, Spain.

\section{Exploring and challenging the network of angiogenesis}

\author{
Raúl Montañez ${ }^{1 *}$, Francisca Sánchez-Jiménez ${ }^{1,2}$, Ana R. Quesada ${ }^{1,2}$ \& Miguel Ángel Medina ${ }^{1,2}$
} 'Department of Molecular Biology and Biochemistry, Faculty of Science, University of Málaga, ${ }^{2}$ Unit 741, CIBER de Enfermedades
Raras (CIBERER), E-29071 Málaga, Spain.

Angiogenesis is one of the hallmarks of cancer and, as such, one of the alternative general targets for anticancer therapy. Since angiogenesis is a complex process involving a high number of interconnected components, a network approach would be a convenient systemic way to analyse responses to directed drug attacks. Herein we show that, although the angiogenic network is easily broken by short combinations of directed attacks, it still remains essentially functional by keeping the global patterns and local efficiency essentially unaltered after these attacks. This is a clear sign of its high robustness and resilience and stresses the need of directed, combined attacks for an effective blockade of the process. The results of this theoretical study could be relevant for the design of new antiangiogenic therapies and the selection of their targets.

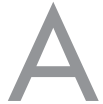

ngiogenesis, the formation of new blood vessels from pre-existing ones, is a hallmark of cancer ${ }^{1}$. Mechanistically, angiogenesis is a very complex process in which several key steps are involved ${ }^{2}$. In fact, when quiescent endothelial cells are activated by some proangiogenic signal, they change their phenotype to become highly proliferative and able to migrate, remodel the surrounding extracellular matrix (ECM) and finally to differentiate to form new vessels. Any of these key steps can be a potential pharmacological target to inhibit angiogenesis and, hence, to treat angiogenesis-dependent diseases ${ }^{3}$. However, the results obtained in the clinical treatment of cancer with approved antiangiogenic compounds show only limited -although significantimprovement. It should be stressed that this first generation of antiangiogenic compounds targets the first step of VEGF biosignaling. As we have previously suggested, since tumor angiogenesis is very complex and involves a number of different cell types, it is possible that multi-target approaches could produce better results $\mathrm{s}^{4,5}$. Therefore, new multi-targeted compounds (or combinations of them) are urgently required to be introduced in the clinical setup.

In a multi-target approach the number of possible unrepeated combinations to explore is $m^{n}$, being $m$ the number of angiogenic elements and $n$ the number of elements to be attacked at every step. The repercussion of such combined approaches will be dependent on the relations among the elements. Therefore, due to the high number of molecular elements contributing to the angiogenic process and the complex relationships among them, the multi-target strategies are far from being under control by using reductionist approaches. Network theory provides a suitable framework for the study of interacting components with a systemic perspective. Herein, we present the utilization of this systemic approach to evaluate the fragility of the angiogenic network against drug attacks.

\section{Results}

The main goal of the present work was to carry out a systemic analysis of the fragility/robustness of the angiogenic process. To achieve this goal, we aimed to build a representative angiogenesis network to be topologically characterized and submitted to directed attacks. Our working hypotheses are: 1 . The angiogenic network is not a random network. 2. According to the previous statement and to the complexity of the process and the different molecules, pathways and cells involved in it, it could be suspected that the angiogenic network is resistant to random attacks. In contrast, the angiogenic network could be easily broken upon several directed attacks. 3 . In spite of this, the angiogenic network could exhibit a high resiliency. A fulfillment of these hypotheses would be consistent with the claimed multi-targeted therapeutical approaches to fight angiogenic diseases.

To start with this research schedule, the first task should be the building of a representative angiogenic network. As mentioned above, the Network of Angiogenesis is defined herein as the collection and integration of proteinprotein interaction, protein modification and transport data from literature, databases and tools for biological data integration. After merging and applying the scoring function to all the initially constructed networks, a 
Table 1 | P-values and percentage of clustering coefficients of each type of networks according to their score values.

\begin{tabular}{lcc} 
GO:0001525 & P-value & \%clustering \\
\hline PPi net high score & $2.38 \times 10^{-67}$ & $42.9 \%$ \\
PPi net medium score & $2.61 \times 10^{-92}$ & $64.1 \%$ \\
PPi net low score & $7.45 \times 10^{-82}$ & $64.9 \%$ \\
Reaction net high score & $4.16 \times 10^{-28}$ & $18.4 \%$ \\
Reaction net medium score & $8.99 \times 10^{-33}$ & $24.5 \%$ \\
Reaction net low score & $7.10 \times 10^{-29}$ & $24.5 \%$ \\
\hline
\end{tabular}

functional analysis with each network was performed by using $\mathrm{BINGO}^{6}$. Table 1 shows that this BINGO analysis pointed to both medium confidence score networks as those incorporating the highest set of angiogenic proteins and with the best p-values, as compared with their respective high and low confidence score counterparts.

Based on this assessment, the union of these two medium confidence score networks was taken as the reference angiogenic network (Figure 1, panel A). Figure 1 (panels B and C) also shows the high and the low confidence score angiogenic networks. The topological characterization of the reference angiogenic network (Figure 1, panel A and Table 2), in general terms, allows to observe that it is enough biased from random network as to think that this network could capture some relevant biological information.

An important signature of some real networks is their degree distribution. If the degree distribution follows a Gaussian
Table 2 | General topological measures from the medium angiogenic network. To compare, the average and standard deviation of 1000 iterations of the null random connection model is included.

\begin{tabular}{lcc} 
Graph properties & Angiogenesis & Random \\
\hline Number of vertices & 337 & $319 \pm 18$ \\
Number of edges & 982 & 982 \\
Avg, Connectivity & 5,82 & $5.81 \pm 0.37$ \\
Avg, Min, Distance & 3,81 & $3,55 \pm 0.01$ \\
Avg, Clustering Coefficient & 0,26 & $0,019 \pm 0.001$ \\
Components & $1 *$ & $1 *$ \\
Assortative Mixing & 0,02 & $-0,003 \pm 0.001$ \\
Global Efficiency & 0,29 & $0,31 \pm 0.06$ \\
Local Efficiency & 0,32 & $0,19 \pm 0.02$ \\
Percolation threshold & 59,02 & $40,27 \pm 3.2$ \\
\hline
\end{tabular}

"Only the biggest connected component was used in order to be able to calculate the averaged minimal distance.

distribution, this is a clue to the random nature of the conformation of this network. On the contrary, if the degree distribution can fit to an asymmetrical and fat tail distribution, this observation points to the fact that some kind of constrains are governing the conformation of the network and that the selected nodes and edges could be a representative selection of the highlighted process. Figure 2 shows that this is the case for the combined representative angiogenic network (panel A), as well as for the angiogenic interactome (panel B) and the angiogenic reaction network (panel C).
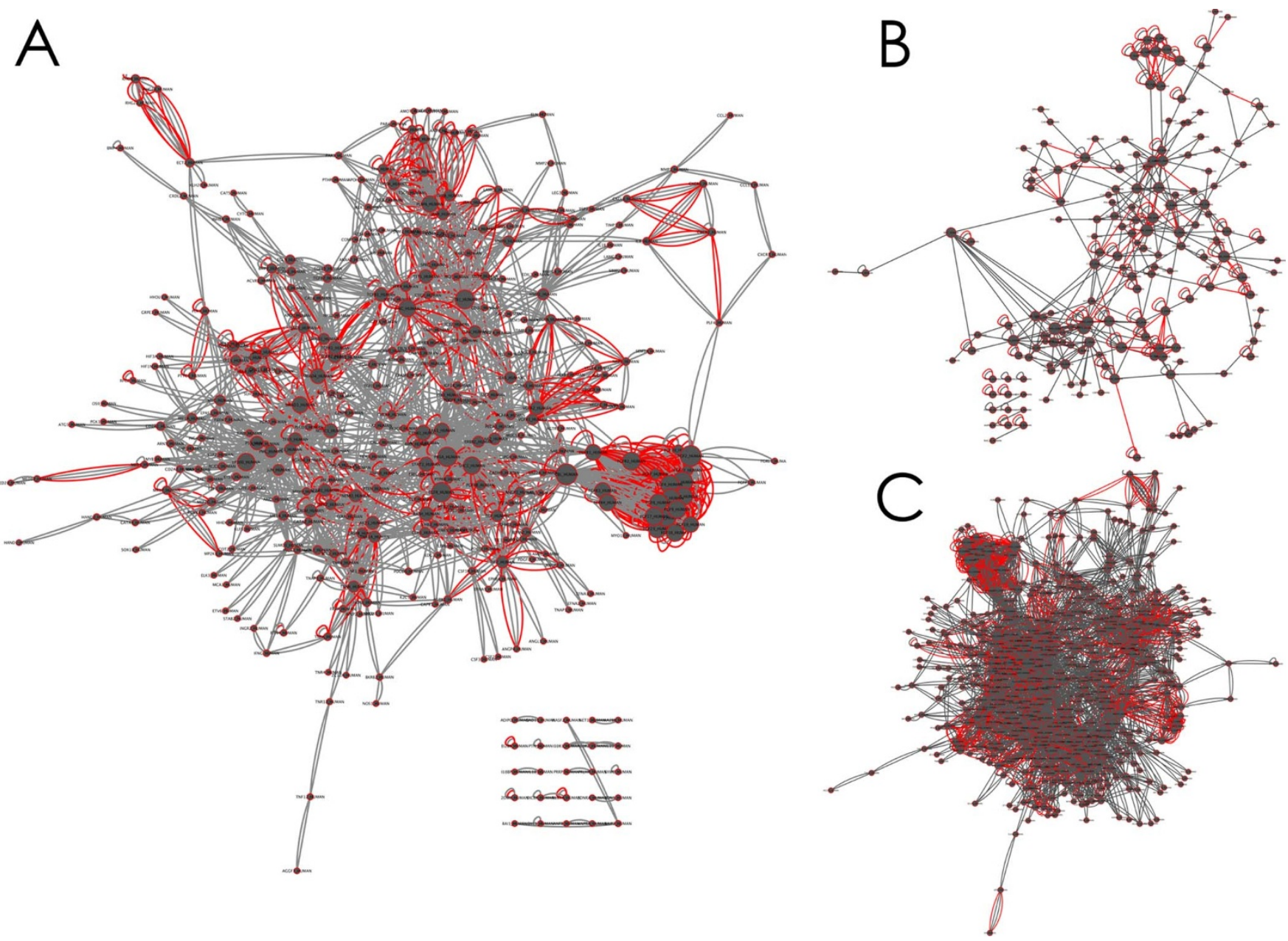

Figure $1 \mid$ The angiogenic network. Combined interaction/reaction networks with different confidence score. (A) The selected representative angiogenic network (that with a medium confidence score). (B) The network with the highest confidence score. (C) The network with the lowest confidence score. 

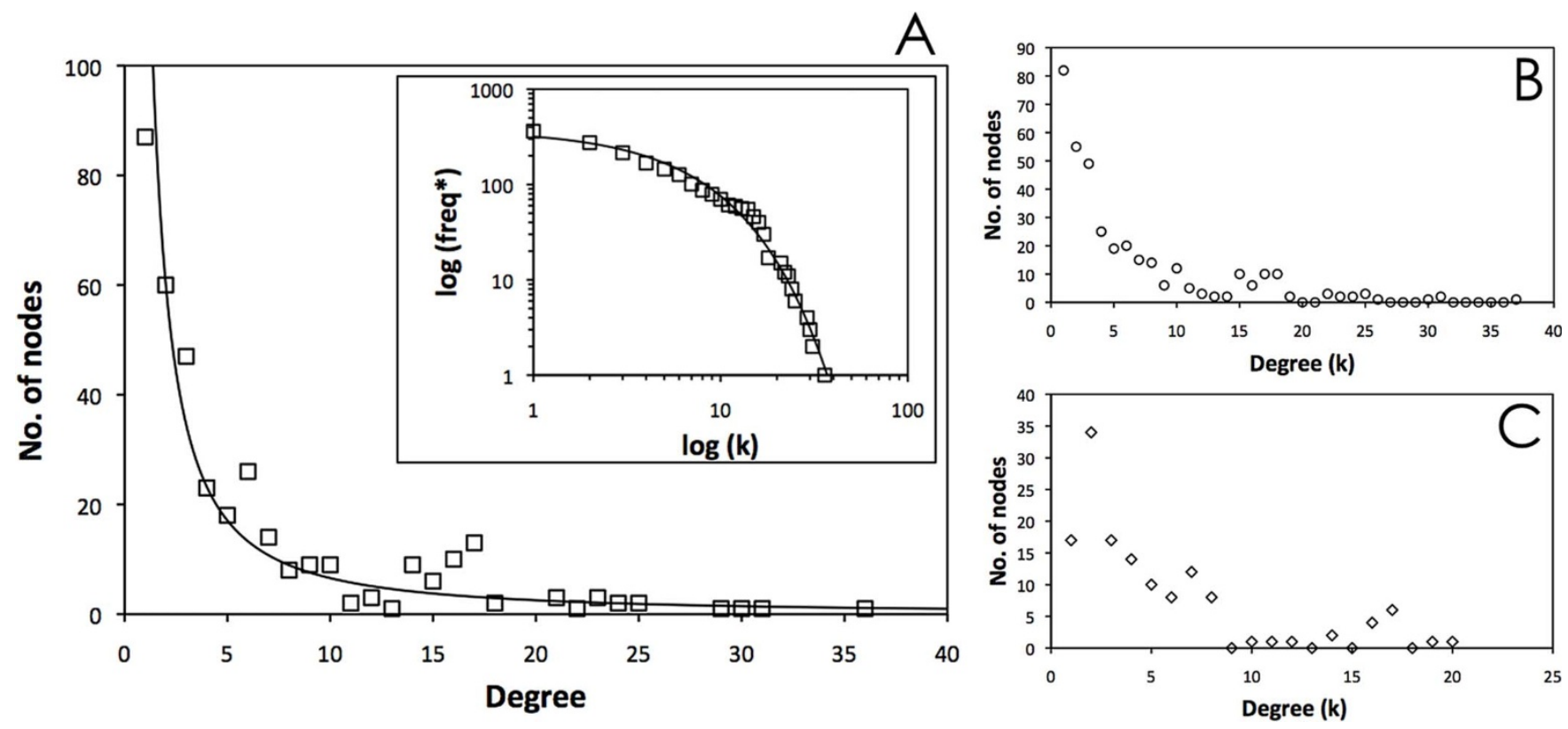

Figure $2 \mid$ Degree distribution of the angiogenic network. (A) Degree distribution of the combined selected angiogenic network and the average values of degree of 1000 random networks (insert). (B) Degree distribution of the angiogenic interactome. (C) Degree distribution of the angiogenic reaction network.

It is known that fat-tail degree distributions confer to the network a high resilience to a random attack but a high sensitivity to a selective node attack ${ }^{7}$. Here we focus on the network tolerance to targeted attack. The fragmentation of real networks after directed attacks has been considered a sign of their fragility ${ }^{7,8}$. As stated in our working hypothesis 2 , the angiogenic network is broken after less directed attacks, as compared to the case of a random network (Figure 3, panel A). However, we must keep in mind that actual cellular protein networks should be expected to be highly fragmented due to the differential expression of proteins according to cell types and environmental conditions. Therefore, at least for protein networks, it could be advisable to make use of the variation in the local efficiency as a better estimator of their fragility/robustness ${ }^{10}$. Here, the local efficiency of the network is analyzed ${ }^{10}$, since it is considered a good estimator of information flow. Local efficiency reveals how efficient the communication is (path length) among the first neighbours of the $n_{i}$ subgraph when this $n_{i}$ is removed (for example, due to drug

A

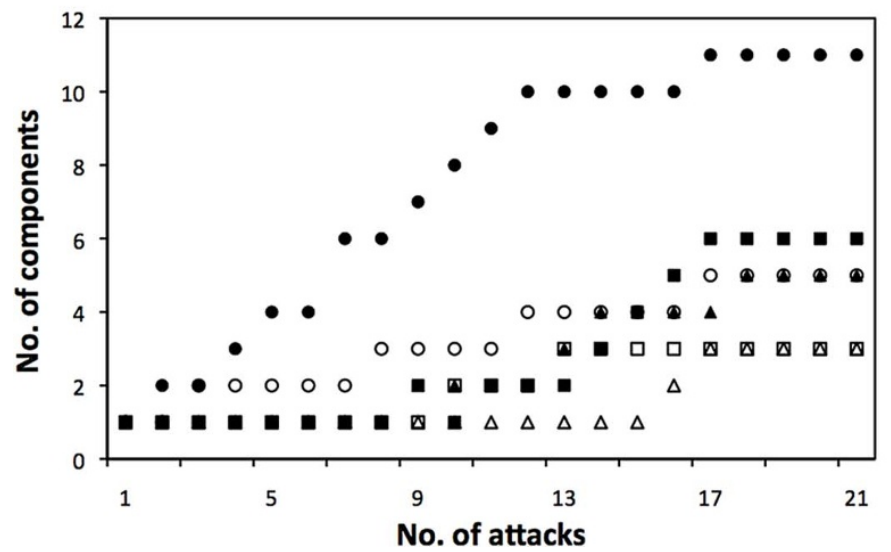

treatments, gene mutations, etc.). Hence, local efficiency is an important coefficient related to vulnerability/robustness ${ }^{10}$. As Figure 3 (panel B) shows, local efficiency of the angiogenic network remains always greater than that corresponding to the random network under the different directed attacks tested, even though the networks were split up in several connected components and this occurred as previously mentioned- after less directed attacks in the angiogenic network (Figure 3, panel A).

In this work, we also suggest that the analysis of the overrepresentation of cross-cluster motifs could be an useful method to validate the functional relationship of connected proteins within a functional and signalling network. Figure 4 shows the 10 different types of motifs than can be found in the network. As we show in Figure 5, motif 5 is the most significantly overrepresented motif.

In addition, the high locality of the angiogenic networks, reflected by the overrepresentation of the most redundant cross-cluster motif, motif 5, (Figure 5), keeps their global patterns and their local

B

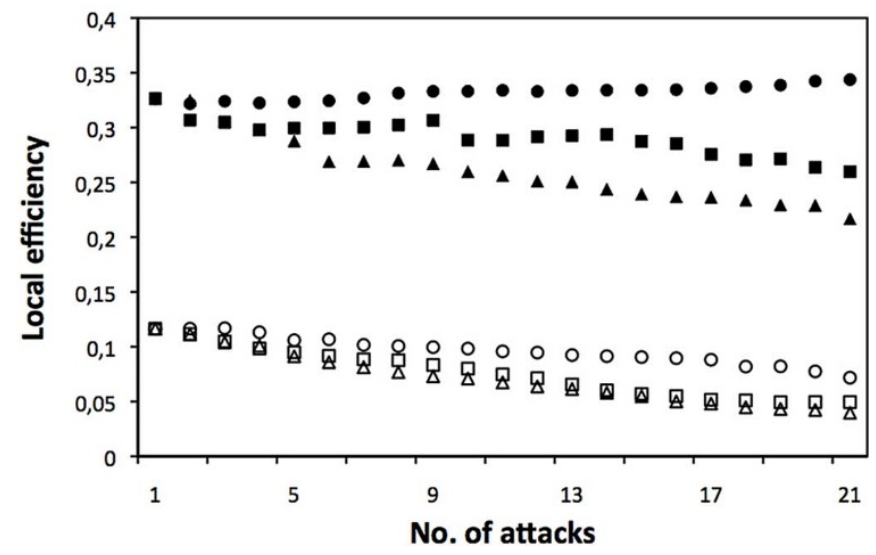

Figure $3 \mid$ Robustness of the angiogenic network. Number of connected components (A) and local efficiency (B) of both real (full symbols) and randomized (void symbols) network after the recursive-targeted attacks RD (recalculated degree distribution, triangles), RB (recalculated betweenness distribution, squares) and RB/D (recalculated betweenness/degree distribution, circles). 

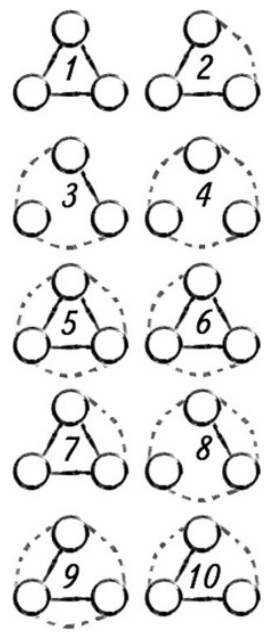

Figure $4 \mid$ Cross-cluster motifs. The set of all the possible combinations of connections for three elements (named cross-cluster motifs along the manuscript). Continuous edges represent physical interactions between proteins and dashed edges represent a common event of protein modification or transport.

efficiency essentially unaltered after a systematic RB/D attack (Figure 5 and Figure 3, panel A).

Although this is not within the aims and scope of the present research work, it should be underscored that the angiogenesis network by us constructed and depicted in Figure 1A maintains a whole array of biologically relevant information making it possible to proceed to a further analysis of its biological insights, as depicted in three selected zooms of Figure 1A shown in Supplementary information as Figures S1, S2 and S3. As an example of further analysis of biological information associated to the angiogenic network, we carried out a simple functional annotation clustering analysis by making use of DAVID Bioinformatics database web tools ${ }^{11}$. Table S1 (in Supplementary material) lists the 213 clusters that the nodes of the angiogenic network form according to such a functional annotation clustering analysis. The clusters are ranked in the order of increasing p-values. Table S2 (in Supplementary material) lists the gene products included in 3 clusters particularly relevant in angiogenesis, namely, clusters 1 (associated to descriptions of “angiogenesis"), 2 (associated to description of cell migration, a key step in angiogenesis) and 9 (associated to description of tube morphogenesis, another key step in angiogenesis).

\section{Discussion}

Mono-targeted antiangiogenic therapies have obtained at most very limited success in the clinical setting ${ }^{3,5}$. The high complexity of the process and of the inter-relationships among the partners have made to claim for requested, new combined, multi-targeted therapeutical approaches ${ }^{4}$. However, simple combinatorial calculations makes it clear that experimental testing of the whole set of possible combinations could be extremely expensive and time-consuming. In this scenario, systemic biocomputational approaches could be useful for a preselection of the best strategies to be experimentally tested. The modern science of networks provides a convenient framework for such a task ${ }^{12}$. Therefore, we decided to build a representative angiogenic network to test our three working hypotheses mentioned above.

The results shown in figures 1-2 and Tables 1-2 clearly rule out the possibility of considering angiogenesis networks as random networks. Therefore, our working hypothesis 1 was confirmed. Furthermore, the topological features of the angiogenic network contribute to its robustness against random attacks (not shown), as stated by working hypothesis 2 . Since the angiogenesis network shows topological properties far away from the properties exhibited by a random network, this makes possible to hypothesize that it emerged under strict evolutionary constraints. In spite of the fact that a fat tail degree distribution has been correlated with a highest vulnerability to target attack $^{7}$, the angiogenic network seems to be robust against the several applied attacks, according to the results obtained on the changes in local efficiency after directed or random attacks (Figure 3, panel B). And this in spite of the fact that the angiogenic network is broken after less directed attacks than random networks (Figure 3, panel A), as expected and stated in our working hypothesis 2 . This observation suggests that the angiogenic network has a singular shape that should be underscored. The reason of this unusual resiliency could be the high level of local connections and organizational redundancy in their overrepresented motifs, as shown in the cross-motif elements exploration. The redundancy in its structure, as a result of the highest densities of motif 5 (Figure 5),

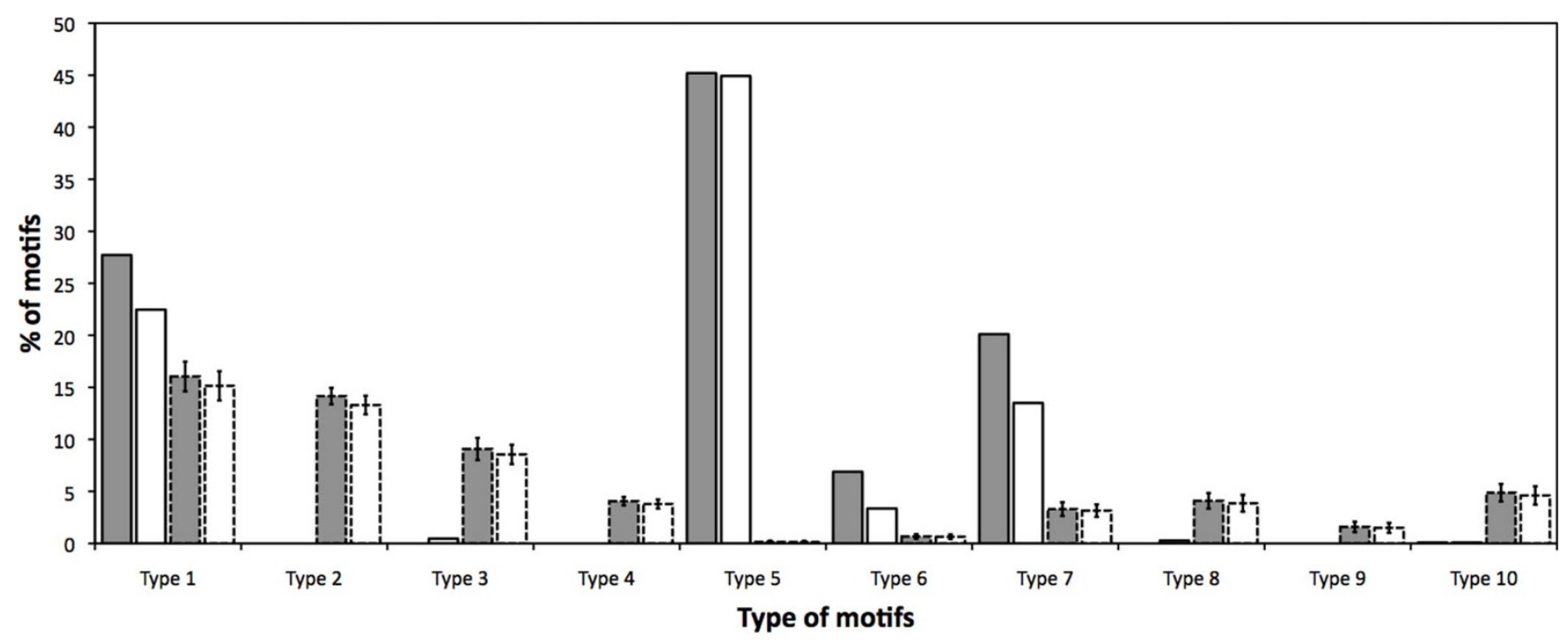

Figure $5 \mid$ Distribution of cross-cluster motifs in the angiogenic network. Percentual representation of the different cross-cluster motifs shown in figure 4 for the selected angiogenic network (solid lines) respect to the random network (dotted lines) before (grey bar) and after (white bar) a selective attack (RD, recalculated degree distribution). In the case of random networks, 1000 independent networks were built and the means \pm SD values are represented. 
maintains the network stable even after ten steps of directed attacks. This observation is relevant, since motif 5 is (among the ten kinds of motifs shown in Figure 4) that with the lowest probability in a completely random null model. The interpretation of this event is that in the angiogenic network the connection among nodes obeys functional requirements (in contrast with a simple random connection), giving priority to those local connections produced as a consequence of the physical interactions, as well as modifications and transport events related with the angiogenic process. Altogether, results shown in Figures 3 and 5 can be interpreted as a clear sign of the high robustness and resilience of the angiogenic networks, provided by their reinforcement of signal transmissions through physics interactions among the involved proteins. These results reinforce our hypothesis 4 and are in agreement with the claimed requirement for multi-targeted therapeutical approaches to fight angiogenesisdependent diseases. Therefore, the angiogenic network reveals fitted and resilient machinery, reflecting the high level of regulation exerted on this network by their channelled cybernetic elements, since these cybernetics elements are involved in the control and coordination of cellular process ${ }^{13,14}$. We are aware that it is obvious that not all the proteins or connections are occurring at the same time and at the same place. This reinforces the idea that more experimental information (in reference to the expression levels at the several states of angiogenesis and the reactions among the elements) is necessary to test which of these motifs are occurring at different moments of the angiogenic process and the real vulnerability of the depicted network.

All in all, compliance with the three starting hypotheses here formulated reinforces the idea that the angiogenesis regulation elements are related as a very resilient system and its resiliency supports the claims for requested multi-targeted anti-angiogenic therapies ${ }^{4,5}$. Therefore, as suggested above, network approach together with the experimental validation could be a useful approach to explore the minimal number of targets and the more essentials elements in this multi-target attack scenario.

Although the main goal of the present work was to carry out a systemic analysis of the fragility/robustness of the angiogenic process, it is also possible to carry out additional analysis to put the angiogenic network in its biological context. Due to the biological relevance of deregulated angiogenesis in cancer progression, a comparison with similar networks of cancer makes sense. When comparing to a general cancer signaling network ${ }^{15}$, several major insights are revealed: i) With its 362 nodes and 1195 edges, the angiogenic network depicted in Figure 1A is much smaller than the cancer signaling network, containing 1397 nodes and 4827 edges. ii) Gene products directly related with the angiogenic switch (such as VEGFs, FGFs and their receptors; see Figure S1 in Supplementary material) or involved in key steps of angiogenesis, such as extracellular matrix remodeling (this is the case of MMPs, urokinase and their inhibitors, TIMPs and PAIs, respectively; see Figure S2 in Supplementary material), endothelial cell migration (as it is the case of the adhesion molecules integrins; see Figures S2 and S3 in Supplementary material) and tube morphogenesis have a key important role (both in terms of their connections and their participation in functional clusters) in the angiogenic network, which is not the case within the network of cancer signaling. iii) The main hub in cancer network p53 remains well connected in the angiogenic network but it is not as relevant in this biological context, as revealed by the middle size of the node representing it in Figure S3 (Supplementary material). Furthermore, the simple functional annotation clustering analysis carried out with DAVID Bioinformatics Resources reveals that the nodes of the angiogenic network depicted in Figure 1A determine 213 functional clusters (listed in Table S1, Supplementary material). As expected, the uppest cluster in the rank of p-values is cluster 1, associated to GO terms IDs 0048514, 0001944, 0001568 and 0001525 with the functional descriptions "blood vessel morphogenesis", "vasculature development", "blood vessel development" and "angiogenesis".
Furthermore, clusters 2-4 are related to endothelial cell migration and cluster 9 is associated to tube morphogenesis, two key steps of the angiogenic process. Table S2 (Supplementary material) lists the whole array of gene products included in clusters 1, 2 and 9, according to their association to each of the GO terms IDs associated to a biological function revealed for each of the mentioned clusters. As expected, among these three clusters, the greatest one is that associated to the overall process of angiogenesis (cluster 1) and the smallest one is that related to the specialized process of tube morphogenesis (cluster 3). The great redundancy of the nodes associated to the different GO terms included in each cluster is also expected. Additional deeper biological function analysis of the angiogenic network is beyond the limits of this work. Further future work in this direction seems warranted.

The high complexity of angiogenesis regulation, controlled by multiple complementary, overlapping and independent pathways, could explain -at least in part- the limited clinical success met by antiangiogenic monotherapies. Combination therapies, acting simultaneously on several pharmacological targets, could help to reach an effective inhibition of tumor angiogenesis. Network analyses such as that presented in this communication reinforce this concept of combination therapy and stress the importance of the selection of targets, since a simple breakdown of the network does not warrant a loss of its efficiency. Future deeper network analyses could throw light upon which targets could be simultaneously inhibited to lead to an effective inhibition of tumor angiogenesis. Such systemic approaches could help to increase the success rate of in vitro and preclinical studies carried out with drug combinations. The expected results could also contribute to the design of new therapeutic strategies for a number of other angiogenic-dependent diseases for which antiangiogenic approaches have already shown benefits.

\section{Methods}

Angiogenic network reconstruction. In the reconstruction of both, physical interactions and common reactions angiogenic networks, several information sources have been merged in order to obtain a more high accuracy and a more reliable set of proteins and relationships. The set of proteins that finally has been considered to be involved in angiogenesis was collected from several sources, such as online available literature ${ }^{4,16-21}$, databases, such as Gene Ontology ${ }^{22}$, Uniprot ${ }^{23}$, APID $^{24}$ or Reactome $^{25}$, computational and experimental tools such as Protein lounge ${ }^{26}$, Ingenuity Pathway ${ }^{27}$ and Agilent Literature search ${ }^{28}$, and commercial experimental sets such as G-arrays (SuperArray). The retrieved information from each of the nine different data resources used was merged in a set of angiogenic nodes. From these sets, their different possible combinations obtained connecting them by union and/or intersection were taken into account in order to build two different kinds of angiogenic networks. Firstly, angiogenic interactomes, making use of the data extracted from those databases containing actual information on protein-protein interaction, namely, Uniprot, APID and Reactome. On the other hand, a second set of angiogenic networks was built connecting nodes sharing a common reaction. After that, each node and interaction was ranked and evaluated according to its redundancy (that is, the number of data sources in which it appears) and the relative reliability of the sources in which it appears, according to the simple following equation: $\mathbf{S}=\sum \mathrm{Ns} \sum \mathrm{Ss}$, where $\mathrm{S}$ is the score value, Ns, refers to the redundancy, that is, the number of data sources in which it appears, and Ss makes reference to the reliability of each event source, using the following simple score: Literature (6), Exp. Tool (5), computational Tools (4), Uniprot (3), Gene Ontology (2), AGIL (1), Reactome (1) and Protein lounge (1). For the next validation step, from each set of interaction and reaction networks we took three key representative networks of the score distribution, namely, the network with the mean score value and the two most external networks in both sides of the score distribution. They were named as high, medium and low score networks, according to their score values.

These six selected angiogenic networks (three interaction networks and three reaction networks) were functionally tested according to the p-values and maximal number of proteins grouped within the GO cellular process term "Angiogenesis" (GO:0001525) using BINGO $^{6}$, a pluging extension of Cytoscape ${ }^{29,30}$. Finally, since both medium score networks exhibited the lowest p-values and the highest percentage of nodes clustered into the angiogenesis class (GO:0001525), they were selected for further analysis.

For the sake of comparison, systematically all the analyses carried out with the selected angiogenic networks were also performed with 1000 random networks.

Measure of the topological properties. The topological properties of the selected graphs were valued using the Nets package algorithm ${ }^{31}$. For a deeper explanation of each measure, see ${ }^{32}$ 
Measurement of the cross-correlation. An important coefficient concerning the topological functionality and collective dynamics of the networks is their locality ${ }^{33}$ For a network with one type of edges, locality is usually evaluated according to the degree of clustering, which can be estimated by the local clustering coefficient ${ }^{34}$ as the probability of a link among neighbors of node i:

$\mathrm{Ci}=\mathrm{P}\{\Delta \mathrm{jk}=1 \mid \Delta \mathrm{ij}=\Delta \mathrm{ik}=1\}$, where $\Delta \mathrm{ij}$ is the adjacency matrix of the network.

The clustering coefficient for networks with two types of edges (e.g., edges of type $\mathrm{P}$ and $\mathrm{R}: \Delta \mathrm{ijP}$ and $\Delta \mathrm{ijR}$ ) can be generalized as the probability of a P-edge between Rneighbors of $\mathrm{i}: \mathrm{Ci}^{\mathrm{P} \mid \mathrm{R}}=\operatorname{Pr}\left\{\Delta \mathrm{jk}^{\mathrm{P}}=1 \mid \Delta \mathrm{ij}^{\mathrm{R}}=\Delta \mathrm{ik}^{\mathrm{R}}=1\right\}$. This is the cross-cluster coefficient measure. Cross-cluster motifs represent an extension of the cross-cluster coefficient measure ${ }^{35}$ and the concept of motif ${ }^{36}$. Here we extend this $\mathrm{Ci}^{\mathrm{G} \mid \mathrm{M}}$ probability to the ten possible combinations (Figure 4):

$$
\begin{aligned}
& \mathrm{C}_{\mathrm{i}}{ }^{1}=\operatorname{Pr}\left\{\Delta \mathrm{jk}^{\mathrm{P}}=1 \mid \Delta \mathrm{ij}^{\mathrm{P}}=\Delta \mathrm{ik}^{\mathrm{P}}=1\right\}, \\
& \mathrm{C}_{\mathrm{i}}{ }^{2}=\operatorname{Pr}\left\{\Delta \mathrm{jk}^{\mathrm{P}}=1 \mid \Delta \mathrm{ij}^{\mathrm{R}}=\Delta \mathrm{ik}^{\mathrm{R}}=1\right\}, \\
& \mathrm{Ci}^{3}=\operatorname{Pr}\left\{\Delta \mathrm{jk}^{\mathrm{R}}=1 \mid \Delta \mathrm{ij}^{\mathrm{P}}=\Delta \mathrm{ik}^{\mathrm{P}}=1\right\}, \\
& \mathrm{Ci}^{4}=\operatorname{Pr}\left\{\Delta \mathrm{jk}^{\mathrm{R}}=1 \mid \Delta \mathrm{ij}^{\mathrm{R}}=\Delta \mathrm{ik}^{\mathrm{R}}=1\right\}, \\
& \mathrm{Ci}^{5}=\operatorname{Pr}\left\{\Delta \mathrm{jk}^{\mathrm{P}}=\Delta \mathrm{ij}^{\mathrm{P}}=\Delta \mathrm{ik}^{\mathrm{P}}=1 \mid \Delta \mathrm{jk}^{\mathrm{R}}=\Delta \mathrm{ij}^{\mathrm{R}}=\Delta \mathrm{ik}^{\mathrm{R}}=1\right\}, \\
& \mathrm{Ci}^{6}=\operatorname{Pr}\left\{\Delta \mathrm{jk}^{\mathrm{P}}=\Delta \mathrm{ij}^{\mathrm{P}}=1 \mid \Delta \mathrm{jk}^{\mathrm{R}}=\Delta \mathrm{ij}^{\mathrm{R}}=\Delta \mathrm{ik}^{\mathrm{R}}=1\right\}, \\
& \mathrm{Ci}^{7}=\operatorname{Pr}\left\{\Delta \mathrm{jk}^{\mathrm{P}}=1 \mid \Delta \mathrm{jk}^{\mathrm{R}}=\Delta \mathrm{ij}^{\mathrm{R}}=\Delta \mathrm{ik}^{\mathrm{R}}=1\right\}, \\
& \mathrm{Ci}^{8}=\operatorname{Pr}\left\{\Delta \mathrm{jk}^{\mathrm{R}}=1 \mid \Delta \mathrm{jk}^{\mathrm{P}}=\Delta \mathrm{ij}^{\mathrm{P}}=\Delta \mathrm{ik}^{\mathrm{P}}=1\right\}, \\
& \mathrm{Ci}^{9}=\operatorname{Pr}\left\{\Delta \mathrm{jk}^{\mathrm{R}}=\Delta \mathrm{ij}^{\mathrm{R}}=1 \mid \Delta \mathrm{jk}^{\mathrm{P}}=\Delta \mathrm{ij}^{\mathrm{P}}=\Delta \mathrm{ik}^{\mathrm{P}}=1\right\}, \\
& \mathrm{Ci}^{10}=\operatorname{Pr}\left\{\Delta \mathrm{jk}^{\mathrm{P}}=\Delta \mathrm{ij}^{\mathrm{P}}=1 \mid \Delta \mathrm{ij}^{\mathrm{R}}=\Delta \mathrm{ik}^{\mathrm{R}}=1\right\},
\end{aligned}
$$

Analysis of the resiliency of the angiogenic networks against node attacks. In order to achieve a deeper understanding of the angiogenesis process and its potential robustness, we performed three kinds of systematic and recurrent attacks against the selected angiogenic networks: (RD) recalculated degree distribution, (RB) recalculated betweenness distribution and $(\mathrm{RB} / \mathrm{D})$ recalculated betweenness / degree distribution. At every step, either the most connected (RD), the most central (RB) or the most bottleneck (RB/D) node of the network was attacked. After the attack, we recalculated to identify the new node in the top of each rank and removed it in the following attack. At each removal step (that is, after each attack), we tested the number of vertices and edges, average connectivity, average minimal distance, average clustering coefficient, size and number of components, global and local efficiency and the percolation threshold as a way to identify when the networks lost their functionality.

Functional annotation clustering analysis. We used DAVID Bioinformatics Resources $^{11}$, available online in http://david.abcc.ncifcrf.gov/

1. Hanahan, D., Bergers, G., and Bergsland, E. Less is more, regularly: metronomic dosing of cytotoxic drugs can target tumor angiogenesis in mice. J Clin Invest 105, 1045-1047 (2000).

2. Muñoz-Chápuli, R., Quesada, A. R., and Medina, M. A. Angiogenesis and signal transduction in endothelial cells. Cell Mol Life Sci 61, 2224-2243 (2004).

3. Quesada, A. R., Muñoz-Chápuli, R., and Medina, M. A. Anti-angiogenic drugs: from bench to clinical trials. Med Res Rev 26, 483-530 (2006).

4. Quesada, A. R., Medina, M. A., and Alba, E. Playing only one instrument may be not enough: limitations and future of the antiangiogenic treatment of cancer. Bioessays 29, 1159-1168 (2007).

5. Quesada, A. R., Medina, M. A., Muñoz-Chápuli, R., and Ponce, A. L. Do not say ever never more: the ins and outs of antiangiogenic therapies. Curr Pharm Des 16, 3932-3957 (2010).

6. Maere, S., Heymans, K., and Kuiper, M. BiNGO: a Cytoscape plugin to assess overrepresentation of gene ontology categories in biological networks. Bioinformatics 21, 3448-3449 (2005).

7. Albert, R., Jeong, H., and Barabasi, A. L., 2000 (unpublished).

8. Crucitti, P., Latora, V., Marchiori, M., and Rapisarda, A. Error and attack tolerance of complex networks. Physica A: Statistical Mechanics and its Applications 340, 388-394 (2004).

9. Han, J. D. J. et al. Evidence for dynamically organized modularity in the yeast proteinñprotein interaction network. Nature 430, 88-93 (2004).

10. Latora, V. and Marchiori, M. Efficient behavior of small-world networks. Physical Review Letters 87, 198701 (2001).

11. Huang, D. W., Sherman, B. T., Lempicki, R. A. Systematic and integrative analysis of large gene lists using DAVID Bioinformatics Resources. Nat Prot 4, 44-57 (2009).

12. Newman, M. E. J., Networks, 1 ed. (Oxford University Press, Oxford, 2010).

13. Mangan, S. and Alon, U. Structure and function of the feed-forward loop network motif. Proc Natl Acad Sci U S A 100, 11980-11985 (2003).
14. Yeger-Lotem, E. et al. Network motifs in integrated cellular networks of transcription-regulation and protein-protein interaction. Proc Natl Acad Sci US A 101, 5934-5939 (2004).

15. Cui, Q. et al. A map of human cancer signaling. Mol Syst Biol 3, 152 (2007).

16. Karamysheva, A. F. Mechanisms of angiogenesis. Biochemistry (Mosc) 73, 751762 (2008).

17. Kerbel, R. S. Tumor angiogenesis. N Engl J Med 358, 2039-2049 (2008).

18. Hillen, F. and Griffioen, A. W. Tumour vascularization: sprouting angiogenesis and beyond. Cancer Metastasis Rev 26, 489-502 (2007).

19. Döme, B. et al. Alternative vascularization mechanisms in cancer: Pathology and therapeutic implications. Am J Pathol 170, 1-15 (2007).

20. Fong, G. H. Mechanisms of adaptive angiogenesis to tissue hypoxia. Angiogenesis 11, 121-140 (2008)

21. Risau, W. Mechanisms of angiogenesis. Nature 386, 671-674 (1997).

22. Ashburner, M. et al. Gene ontology: tool for the unification of biology. The Gene Ontology Consortium. Nat Genet 25, 25-29 (2000).

23. Consortium, UniProt The Universal Protein Resource (UniProt) in 2010. Nucleic Acids Res 38, D142-148 (2010).

24. Prieto, C. and De Las Rivas, J. APID: Agile Protein Interaction DataAnalyzer. Nucleic Acids Res 34, W298-302 (2006).

25. Joshi-Tope, G. et al. Reactome: a knowledgebase of biological pathways. Nucleic Acids Res 33, D428-432 (2005)

26. ProteinLounge, Available at http://www.proteinlounge.com/.

27. Ganter, B. and Giroux, C. N. Emerging applications of network and pathway analysis in drug discovery and development. Curr Opin Drug Discov Devel 11, 8694 (2008).

28. Vailaya, A. et al. An architecture for biological information extraction and representation. Bioinformatics 21, 430-438 (2005).

29. Cline, M. S. et al. Integration of biological networks and gene expression data using Cytoscape. Nat Protoc 2, 2366-2382 (2007).

30. Shannon, P. et al. Cytoscape: a software environment for integrated models of biomolecular interaction networks. Genome Res 13, 2498-2504 (2003).

31. Fernández, P, Nets, Available at http://www.lsi.upc.edu/ pfernandez/softwarenetworks.html, (2007).

32. Newman, M. E. J. The structure and function of complex networks. SIAM review 45, 167-256 (2003)

33. Watts, D. J. and Strogatz, S. H. Collective dynamics of 'small-world' networks. Nature 393, 440-442 (1998).

34. Dorogovtsev, S. N. and Mendes, J. F. F. The shortest path to complex networks. Arxiv preprint cond-mat/0404593 (2004).

35. Spirin, V., Gelfand, M. S., Mironov, A. A., and Mirny, L. A. A metabolic network in the evolutionary context: multiscale structure and modularity. Proc Natl Acad Sci U S A 103, 8774-8779 (2006).

36. Milo, R. et al. Network motifs: simple building blocks of complex networks. Science 298, 824-827 (2002).

\section{Acknowledgements}

Our experimental work is supported by grants PS09/02216 and SAF 2011-26518 (Spanish Ministry of Science and Innovation), and PIE P08-CTS-3759, CVI-6585 and funds from group BIO-267 (Andalusian Government). The "CIBER de Enfermedades Raras" is an initiative from the ISCIII (Spain). The funders had no role in study design, data collection and analysis, decision to publish, or preparation of the manuscript.

\section{Author contributions}

RM and MAM designed research; RM performed research; RM, ARQ, FSJ and MAM analyzed and discussed data; MAM wrote the paper; and RM, ARQ, FSJ and MAM revised the manuscript draft and contributed with suggestions to the final version of the paper.

\section{Additional information}

Supplementary Information accompanies this paper at http://www.nature.com/ scientificreports

Competing financial interests: The authors have declared no competing financial interests.

License: This work is licensed under a Creative Commons

Attribution-NonCommercial-NoDerivative Works 3.0 Unported License. To view a copy of this license, visit http://creativecommons.org/licenses/by-nc-nd/3.0/

How to cite this article: Montañez, R., Sánchez-Jiménez, Quesada, A.R. \& Medina, M.Á. Exploring and challenging the network of angiogenesis. Sci. Rep. 1, 61; DOI:10.1038/ srep00061 (2011). 